\title{
VOLTAMETRIA DE ONDA QUADRADA. PRIMEIRA PARTE: ASPECTOS TEÓRICOS
}

Djenaine de Souza, Sergio A. S. Machado* e Luis A. Avaca

Instituto de Química de São Carlos, Universidade de São Paulo, CP 780, 13560-970 São Carlos - SP

Recebido em 23/1/02; aceito em 22/7/02

\begin{abstract}
SQUARE WAVE VOLTAMMETRY. PART I: THEORETICAL ASPECTS. The theoretical aspects of square wave voltammetry were discussed. Reversible, irreversible and quase-reversible electrode reactions were analyzed and the correlations between parameters like frequency, period, square wave potential and amplitude were showed. In this way, diagnostic relationships allow to characterize the electrode process. The analytical applications were discussed in base of the increment in the analytical response (current) due to the characteristics of the developed equations and the unique mode of collecting the electrode response, i.e., the direct and reverse signals. Finally, recent advances in the basic theory, as the applications to the hydrodynamic electrode and the ultramicroelectrode were also analyzed, and the multiple pulses square wave voltammetry was also introduced.
\end{abstract}

Keywords: square wave voltammetry; electroanalysis; pulse electroanalytical techniques.

\section{INTRODUÇÃO}

Um conjunto de técnicas voltamétricas de análises, conhecido pela denominação abrangente de técnicas eletroanalíticas, está sendo cada vez mais utilizado em áreas do conhecimento tão distintas como medicina, bioquímica, biologia molecular, química ambiental e físico-química ${ }^{1}$, com o objetivo de se obter informações fundamentais sobre propriedades intrínsecas das substâncias. Estudos de processos de oxidação e redução em vários meios, de adsorção em superfícies e de mecanismo de transferência de elétrons, inclusive com a utilização de eletrodos modificados, exemplificam algumas das numerosas aplicações atuais das chamadas técnicas eletroanalíticas ${ }^{2}$.

Uma de suas mais importantes características relaciona-se com o fato destas técnicas possibilitarem o estabelecimento de relações diretas entre a concentração do analito e alguma propriedade elétrica como corrente, potencial, condutividade, resistência ou carga. Como as medidas destas propriedades são facilmente acessíveis experimentalmente, as técnicas eletroanalíticas são adequadamente utilizadas na quantificação de espécies de interesse nas diferentes áreas de estudo.

Uma grande vantagem destas técnicas consiste na possibilidade da medida ser realizada diretamente na amostra sem necessidade de etapas de pré-purificações ou de separações prévias, além de tornar possível a análise de materiais coloridos ou amostras contendo partículas sólidas dispersas ${ }^{3}$.

Estas vantagens, aliadas ao curto tempo na realização das análises, ao baixo custo da instrumentação e dos materiais utilizados, se comparados às técnicas cromatográficas e espectroscópicas, e à baixa sensibilidade que as técnicas eletroanalíticas apresentam em relação à presença de interferentes, fizeram com que elas sejam cada vez mais intensamente utilizadas. Sua crescente importância levou ao desenvolvimento de técnicas cada vez mais sensíveis às espécies em estudo, algumas inclusive com limites de detecção tão baixos que já podem ser comparados aos das técnicas tradicionais utilizadas na análise de compostos orgânicos e inorgânicos em matrizes ambientais, biológicas e em alimentos.

*e-mail: sasmach@iqsc.sc.usp.br

\section{Técnicas de Pulso}

O desenvolvimento de técnicas eletroanalíticas deve-se, principalmente, à viabilização da instrumentação eletroquímica alcançada com o avanço tecnológico resultante da evolução da informática. A possibilidade de interfaciamento com equipamentos eletroquímicos para o controle digital da perturbação imposta ao eletrodo de trabalho, assim como da medida do sinal resultante, possibilitou o desenvolvimento das técnicas voltamétricas, em especial das técnicas de pulso que, na década de 50, começaram a substituir as técnicas polarográficas até então utilizadas ${ }^{4}$.

As técnicas de pulso são baseadas na cronoamperometria, ou seja, na medida da corrente elétrica em função do tempo de aplicação de um determinado pulso de potencial. As características da corrente medida estão relacionadas tanto com a largura do pulso quanto com o degrau de potencial que é aplicado no eletrodo para promover o processo faradaico ${ }^{5}$.

Nas técnicas de pulso, a perturbação do potencial do eletrodo não é uma função linear do tempo do experimento, sendo que a sistemática para a variação de potencial segue uma seqüência de pulsos de potenciais, cujas respostas de corrente obtidas dependem de como estes pulsos são aplicados. Exatamente a diferença na maneira de aplicar os pulsos de potencial é que define as características básicas de cada uma destas técnicas ${ }^{6}$.

Desta forma, a voltametria de pulso normal é baseada em medidas de corrente originadas por uma programação de potencial composta por uma sequiência de pulsos de potencial, com tempo de duração constante e amplitudes crescentes, sobreposta a um potencial de base constante. Neste potencial constante não ocorre nenhum processo faradaico sendo observada apenas uma corrente residual, causada pela presença de oxigênio dissolvido ou outras impurezas eletroativas que porventura estejam presentes em solução, e uma corrente capacitiva que no momento de aplicação do pulso de potencial é, praticamente, constante. O aumento do potencial do eletrodo causado por este pulso, oxida e/ou reduz o analito gerando uma corrente faradaica, que rapidamente decresce até o seu valor mínimo (difusional), característico para cada valor do potencial de pulso (amplitude). Este procedimento é repetido, com pulsos de valores crescentes de potencial, e as correntes geradas são coletadas, após um dado tempo $\tau$ da aplicação do pulso, na forma de uma onda sigmoidal, 
dependente da concentração do analito em solução ${ }^{7}$.

Já na voltametria de pulso diferencial, pulsos de igual amplitude são aplicados sobre uma rampa linear de potencial, a corrente é medida antes do pulso ser aplicado e no final do pulso. Estas correntes são subtraídas, já que a primeira é a contribuição da corrente capacitiva e a segunda é a contribuição da corrente faradaica e, então, graficadas contra o potencial da rampa linear, gerando um voltamograma de pulso diferencial, com a forma de uma curva gaussiana. Esta técnica é mais sensível que a de pulso normal, isto porque ela possibilita a minimização da contribuição da corrente capacitiva no sinal obtido (pois a corrente capacitiva não depende da concentração da espécie em estudo).

A voltametria de pulso normal e diferencial é uma mistura das técnicas de pulso normal e diferencial, apresentando modos alternados de aplicação de pulsos de potencial. A medida inicia-se em um potencial onde não há processo faradaico. O primeiro par de pulsos consiste em um pulso de potencial para um valor $\mathrm{E}_{1}$, seguido por um pequeno pulso diferencial $\Delta \mathrm{E}$, para o valor $\mathrm{E}_{2}$. O potencial é, então, restabelecido ao valor do potencial inicial e os pulsos seguintes são semelhantes aos iniciais, porém, em sentido inverso, $-\Delta \mathrm{E}$. Nesta técnica há uma elevada sensibilidade a pequenos valores de corrente e grande diferenciação das correntes faradaica e capacitiva.

A voltametria de escada ("staircase") envolve uma variação de potencial na forma de uma escada, com os parâmetros sempre fixos, sendo a altura do degrau de potencial $10 \mathrm{mV}$ ou menor e a largura de até $17 \mathrm{~ms}$. A corrente é medida ao final de cada degrau, onde a corrente capacitiva já sofreu um decaimento máximo, considerando-se o intervalo de tempo de aplicação do pulso de potencial. $\mathrm{O}$ voltamograma resultante é semelhante àquele obtido por voltametria de varredura linear, porém com uma diminuição considerável na contribuição da componente de corrente capacitiva, e um valor máximo para a corrente faradaica.

Em todas estas técnicas, como a corrente capacitiva é proporcional a e ${ }^{-t / R C}$, onde $t$ é o tempo, $\mathrm{R}$ a resistência da solução e $\mathrm{C}$ a capacitância da dupla camada, e a corrente faradaica é aproximadamente proporcional a $\mathrm{t}^{-1 / 2}$, o decaimento da primeira, após a aplicação do pulso de potencial, é muito mais rápido que o da última. Assim, as medidas de corrente somente são realizadas após a contribuição da corrente capacitiva ter se minimizado. A Figura 1 apresenta as dependências das correntes faradaica e capacitiva com relação ao tempo e o ponto onde a medida de corrente é realizada, considerando-se as técnicas de pulso. Em todas estas técnicas, a corrente é medida em uma fração de tempo fixa chamada de período $(\tau)$.

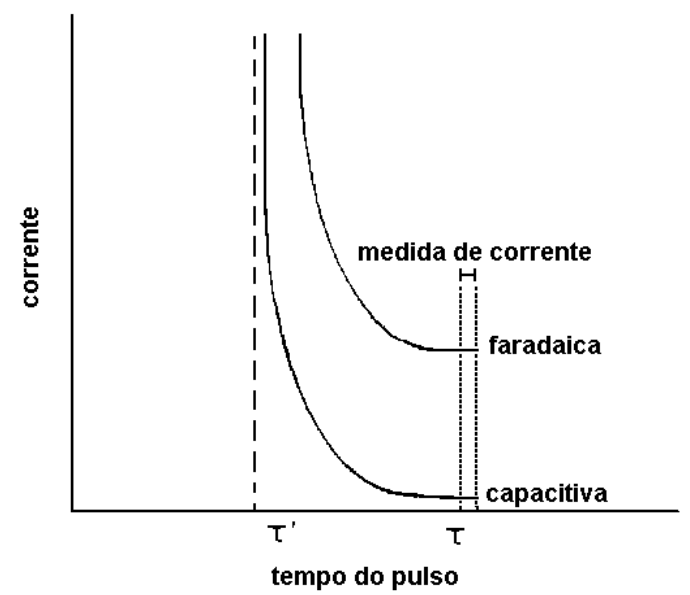

Figura 1. Variação da corrente faradaica e corrente capacitiva com o tempo, em técnicas de pulso
A voltametria de onda quadrada (SWV), do inglês "Square Wave Voltammetry", é uma das técnicas voltamétricas de pulso mais rápidas e sensíveis. Os limites de detecção podem ser comparados aos das técnicas cromatográficas e espectroscópicas. Além disso, a análise dos parâmetros característicos desta técnica também possibilita a avaliação cinética e mecanística do processo eletródico em estudo.

Desta forma, o objetivo desta revisão é mostrar uma discussão da técnica de voltametria de onda quadrada, principalmente em seus aspectos históricos e gerais, assim como apresentar os modelos teóricos desenvolvidos para a aplicação em eletrodos convencionais e eletrodos de mercúrio.

Em revisão posterior, será apresentado um levantamento bibliográfico acerca da aplicação da SWV nos mais diversos meios, dando atenção especial aos trabalhos desenvolvidos no GMEME (Grupo de Materiais Eletroquímicos e Métodos Eletroanalíticos), que utilizam a SWV para estudos analíticos e mecanísticos da redução eletroquímica de alguns pesticidas. Por isto, neste trabalho, citaremos apenas alguns trabalhos relevantes para a aplicação da SWV e seus respectivos modelos teóricos.

\section{Voltametria de onda quadrada: aspectos históricos}

Em 1953, Geoffery Barker ${ }^{8}$ e colaboradores estudavam uma maneira de compensar a corrente capacitiva residual obtida nas análises polarográficas. Para isto, o eletrodo de trabalho era perturbado pela aplicação de uma programação de potenciais na forma de uma onda simétrica com frequiência de $225 \mathrm{~Hz}$ sobreposta a uma rampa de potencial com variação lenta. Esta programação de potenciais foi aplicada a um eletrodo gotejante de mercúrio (EGM), onde a corrente capacitiva era medida ao final da vida de cada gota, a 1,94 milisegundos após cada mudança de potencial da onda quadrada. O sinal final era definido como sendo a diferença das correntes obtidas entre cada semi-ciclo. Esta técnica foi definida como polarografia de onda quadrada.

Para eles, o perfil sigmoidal obtido inicialmente para a polarografia de onda quadrada era semelhante àquele obtido por polarografia de corrente direta. Numa etapa posterior, Barker realizou uma derivação deste perfil, obtendo um pico simétrico onde a intensidade de corrente de pico era proporcional à concentração da espécie eletroativa, o que possibilitava sua utilização na análise de traços.

Contudo, a técnica, como desenvolvida por Barker, possuía uma sensibilidade limitada pela reversibilidade do sistema e, principalmente, pelos ruídos originários de várias fontes e que o capilar de mercúrio juntamente com a coluna captavam e assim, influenciavam intensamente nas respostas de corrente. Para resolver este problema, ele optou por modificar a freqüência de aplicação da onda quadrada, assim como utilizar uma variação mais rápida na rampa de potencial, de maneira a obter uma resposta de corrente-potencial otimizada, ampliando o limite de detecção da metodologia. Porém, os ruídos externos que eram intensificados pela utilização do capilar ainda influenciavam de maneira considerável nas análises.

Por quinze anos, poucos trabalhos foram realizados utilizando a polarografia de onda quadrada, nesta forma inicial, como desenvolvida por Barker e colaboradores. Em 1969, Ramaley e Krause ${ }^{9,10}$ utilizaram eletrodos estacionários, eliminando assim a influência dos ruídos intensificados pelo uso do capilar de mercúrio. Além disso, eles substituíram a rampa linear de potencial por uma variação na forma de escada. Desta maneira, surgiu a voltametria de onda quadrada (SWV) que utilizava uma variação de potencial na forma de onda, aliada a uma rampa de potencial na forma de escada, gerando um pico simétrico que poderia ser utilizado com sucesso para determinações analíticas. 
A Figura 2 apresenta o esquema básico para a SWV da maneira como foi proposta por Ramaley e Krause, onde:

1) representa a aplicação de potencial na forma de onda quadrada;

2) a variação do potencial na forma de uma escada, neste caso considerando uma variação de potenciais para valores negativos;

3) onda quadrada aliada à variação na forma de escada;

4) forma da onda de corrente total, onde $\mathrm{E}<<\mathrm{E}_{\mathrm{p}}$ corresponde a potenciais menos negativos que o potencial de pico, $\mathrm{E}=\mathrm{E}_{\mathrm{p}}$ ao potencial de pico e $\mathrm{E} \gg \mathrm{E}_{\mathrm{p}}$ corresponde a potenciais mais negativos que o potencial de pico;

5) medidas de corrente, antes, no pico e após o pico;

6) corrente diferencial, onde antes do potencial de pico a resultante é zero (as correntes direta e reversa são nulas), no potencial de pico assume o valor máximo $\left(I_{d}-I_{r}\right)$ e após o pico a corrente total é nula, pois as duas correntes difusionais possuem o mesmo sinal e

7) corrente total derivada.

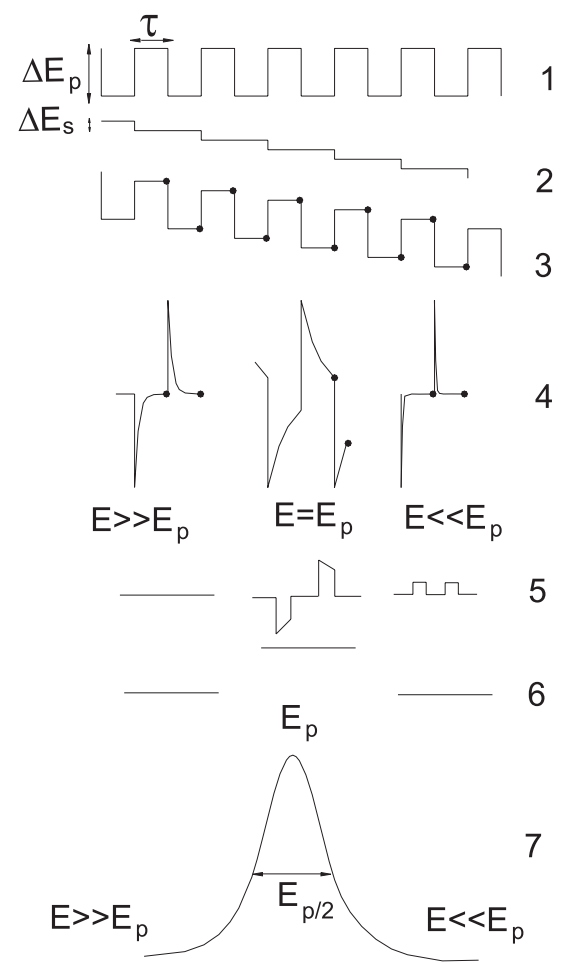

Figura 2. Representação esquemática da voltametria de onda quadrada, onde: 1) potencial na forma de onda; 2) escada de potencial; 3) forma de aplicação do potencial na SWV; 4) forma da onda da corrente; 5) sinal da corrente; 6) corrente diferencial e 7) corrente total

Ramaley e Krause empregaram amplitudes no intervalo de 1,25 a $25 \mathrm{mV}$, freqüências de $100 \mathrm{~Hz}$, e a largura do degrau de potencial variando de $0,025 \mathrm{mV}$ a $0,5 \mathrm{mV}$ para a onda de potencial. Os resultados eram comparáveis àqueles obtidos por Barker.

Da maneira como utilizada por Ramaley e Krause ${ }^{9}$, a medida de corrente era realizada próxima ao centro do pulso e as varreduras de potenciais eram limitadas para pequenos valores de amplitude e, conseqüentemente, baixas velocidades de varreduras, o que limitava a sua aplicação pois provocava uma perda de sensibilidade.

Em 1977 Christie, Turner e Osteryoung ${ }^{11,12}$ analisaram estas limitações e chegaram ao modelo atual da SWV, onde a medida de corrente é realizada com velocidades de varredura maiores que
$100 \mathrm{mV} \mathrm{s}^{-1}$, e as medidas de corrente sendo realizadas apenas ao final do pulso de potencial, onde a magnitude da corrente capacitiva já está minimizada. Esta metodologia possibilitou uma sensível melhora na sensibilidade da técnica, tornando mais viável a sua aplicação para análises. A forma de aplicação de potencial difere superficialmente da forma proposta por Ramaley e Krause, e o tratamento dado por eles possibilita a utilização de eletrodos não-planares, o que não foi considerado anteriormente.

Atualmente na SWV, a forma da curva de corrente-potencial é proveniente da aplicação de potenciais de altura $\Delta E_{s}$ (amplitude do pulso de potencial), que variam de acordo com uma escada de potencial com largura $a$ (amplitude do pulso de potencial) e duração $\tau$ (período). Na curva de potencial-tempo, a largura do pulso $(\tau / 2)$ é chamada $t$ e a freqüência de aplicação dos pulsos é chamada de $f$ e é dada por $(1 / t)$. As correntes elétricas são medidas ao final dos pulsos diretos e reversos e o sinal é obtido como uma intensidade da corrente resultante $(\Delta I)$ de forma diferencial, e apresenta excelente sensibilidade e alta rejeição a correntes capacitivas. Esta medida precede um tempo inicial $\left(t_{i}\right)$ onde o eletrodo de trabalho é polarizado a um potencial onde a reação redox não ocorre.

A Figura 3 apresenta um detalhamento da forma de aplicação do potencial na SWV, já introduzido na Figura 2 com a definição dos parâmetros utilizados, enquanto que a Figura 4 apresenta os voltamogramas teóricos associados a: (1) um sistema reversível e (2) um sistema irreversível, com a separação observada das correntes direta, inversa e resultante (conforme indicado na Figura), sendo que ambos possuem perfis voltamétricos semelhantes aos que eram obtidos na polarografia de onda quadrada.

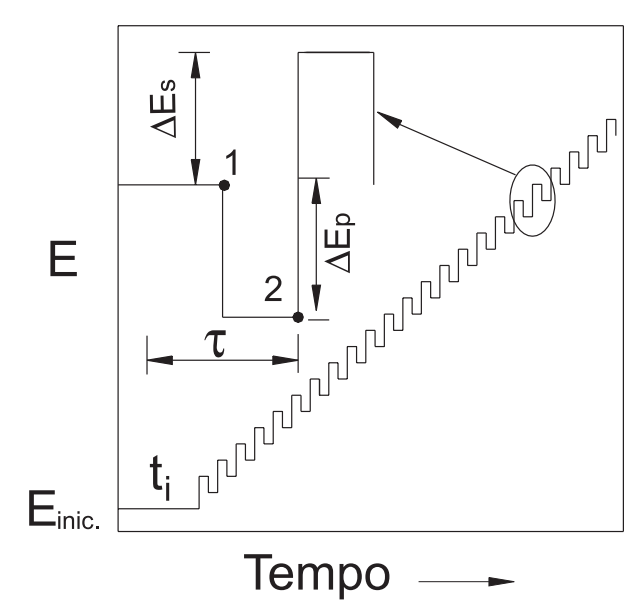

Figura 3. Forma de aplicação do potencial na voltametria de onda quadrada

As curvas de corrente-potencial apresentam perfil bem definido e são, geralmente, simétricas, isto porque as correntes são medidas somente no final de cada semiperíodo e as variações na altura e na largura do pulso de potencial são sempre constantes, para um determinado intervalo de potenciais.

\section{Desenvolvimento de modelos teóricos para a aplicação da voltametria de onda quadrada}

Os modelos utilizados no estudo de espécies eletroativas utilizando a SWV foram basicamente desenvolvidos por dois grupos de pesquisas, o grupo de Janet Osteryoung em Nova York, e o grupo de Milivoj Lovrić na Iugoslávia. A utilização de programas computacionais capazes de simular o comportamento químico para 

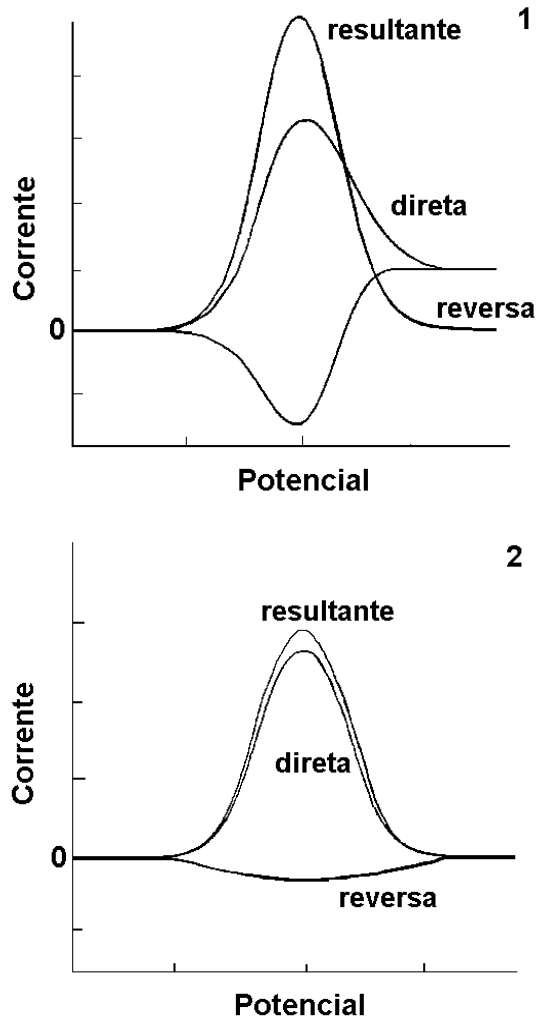

Figura 4. Voltamogramas esquemáticos de onda quadrada onde 1) representa um processo redox de um sistema reversivel e 2) de um sistema irreversivel

sistemas reversíveis, quase-reversíveis e irreversíveis, possibilitou a estes dois grupos desenvolverem toda a teoria da voltametria de onda quadrada, a qual pode ser utilizada no estudo analítico e na obtenção de dados relacionados à cinética e mecanismo de reações químicas, sob as mais variadas condições.

A adsorção de reagentes ou produtos em qualquer reação redox pode causar um aumento significativo nas respostas da SWV, quando comparadas àquelas obtidas por outras técnicas de pulso, especialmente se o sistema é irreversível ou quase-reversível ${ }^{13}$.

Reações redox totalmente irreversíveis são muito importantes em eletroanálise pois muitos compostos orgânicos, normalmente contendo anéis aromáticos homocíclicos ou heterocíclicos, com grupos ligantes eletroativos sofrem reduções totalmente irreversíveis devido à baixa velocidade de transferência de carga, ou, muitas vezes por inativação rápida do produto de redução ${ }^{13}$.

Uma avaliação das respostas voltamétricas obtidas com a SWV, mostra que, se uma reação redox ocorre a partir de um reagente dissolvido, a corrente faradaica decresce muito mais lentamente que a corrente capacitiva. Assim, estas duas componentes podem ser separadas, se a medida é realizada ao final de cada pulso. No entanto, se a reação redox ocorre a partir de um reagente adsorvido, a relação corrente faradaica-tempo depende do grau de reversibilidade da reação e também do potencial do eletrodo de trabalho ${ }^{14}$. Esta foi uma das mais importantes considerações feitas por Osteryoung e Lovrić no desenvolvimento dos modelos para a SWV, além disso, eles consideraram os seguintes esquemas de reações:

(1) Adsorção do reagente

$\mathrm{A} \stackrel{\mathrm{K}_{1}}{\rightleftharpoons} \Gamma_{\mathrm{A}}+\mathrm{e}^{-} \rightleftharpoons \mathrm{B}$
(2) Adsorção do reagente e do produto

$A \stackrel{K_{1}}{\rightleftharpoons} \Gamma_{A}+e^{-} \stackrel{K_{2}}{\rightleftharpoons} \Gamma_{B} \rightleftharpoons B$

(3) Reação redox simples, sem adsorção

$A+e^{-} \rightleftharpoons B$

onde $\Gamma_{\mathrm{A}}$ e $\Gamma_{\mathrm{B}}$ representam os excessos superficiais de reagente e produto adsorvido, respectivamente.

Tanto Osteryoung quanto Lovrić consideraram que a adsorção do produto e reagente surge a partir de uma difusão linear, com o coeficiente de transferência de massa aproximadamente planar $^{14}$, seguindo as Leis de Fick.

Sendo assim, as reações apresentadas nos esquemas (A) e (B) foram matematicamente representadas por um sistema de equações diferenciais, definidas pela $2^{\underline{a}}$ Lei de Fick:

$\frac{\partial A}{\partial t}=D_{A}\left(\frac{\partial^{2} A}{\partial x^{2}}\right) \quad \frac{\partial B}{\partial t}=D_{B}\left(\frac{\partial^{2} B}{\partial x^{2}}\right)$

onde $D_{\mathrm{A}}$ e $D_{\mathrm{B}}$ são os coeficientes de difusão de reagente e produto, respectivamente, e $x$ é a distância da espécie eletroativa até a superfície do eletrodo de trabalho.

A solução para estas equações diferenciais levou em consideração as seguintes condições de contorno:

$t=0, x \geq 0 ; A=A^{*}, B=0$

$\Gamma_{A}=0$

$\Gamma_{B}=0$

$t>0, x \rightarrow \infty: A \rightarrow A^{*}, B \rightarrow 0$

$x=0: A_{x=0}=K_{1} \Gamma_{A}$

$B_{x=0}=K_{2} \Gamma_{B}$

somente para o esquema (B) e somente para o esquema (A)

somente para o esquema (A) somente para o esquema (B)

Além das Leis de Fick, o tratamento matemático leva em consideração a Lei de Faraday, que relaciona densidade de corrente em função da quantidade de massa transferida para a superfície do eletrodo de trabalho. Sendo assim, soluções para as equações diferenciais apresentadas inicialmente são:

$\begin{array}{lr}D_{A}(\partial A / \partial x)=d \Gamma_{A} / d t+i / n F q & \text { para os esquemas (A) e (B) } \\ D_{B}(\partial B / \partial x)=-i / n F q & \text { para o esquema (A) } \\ D_{B}(\partial B / \partial x)=d \Gamma_{B} / d t-i / n F q & \text { para o esquema (B) }\end{array}$

$i / n F q=k_{r} \exp (-\alpha \gamma)\left(\Gamma_{A}-B_{x=0} \exp (\gamma)\right) \quad$ para o esquema (A) $i / n F q=k_{r} \exp (-\alpha \gamma)\left(\Gamma_{A}-\Gamma_{B} \exp (\gamma)\right) \quad$ para o esquema (B)

onde $i$ é a densidade de corrente, $q$ a área do eletrodo, $\gamma=n F\left(E^{0}-\right.$ $\left.E_{\Gamma / \mathrm{B}}\right) R T$ para o esquema (A) e $\gamma=n F\left(E^{0}-E_{\Gamma_{1} / \Gamma_{\mathrm{B}}}\right) R T$ para o esquema (B), $D_{A}$ e $D_{B}$ são os coeficientes de difusão, $k_{r}$ é a constante de velocidade da reação, $A_{x=0}$ e $B_{x=0}$ são as concentrações de reagente e produto próximos à superfície do eletrodo, $A^{*}$ é a concentração do reagente no seio da solução, $\Gamma_{\mathrm{A}}$ e $\Gamma_{\mathrm{B}}$ são as concentrações do reagente e produto adsorvido, respectivamente, $\alpha$ é o coeficiente de transferência eletrônica, $K_{1}$ e $K_{2}$ são as constantes de adsorção, $n$ o número de elétrons, $R$ a constante dos gases, $F$ a constante de Faraday, e $T$ a temperatura ${ }^{15}$.

As relações entre os parâmetros do sistema (potencial padrão, constante de velocidade e coeficiente de transferência de carga) e os parâmetros voltamétricos (altura, posição e largura do pico voltamétrico) foram considerados para o desenvolvimento e resolução das equações diferenciais apresentadas.

A solução para as equações diferenciais desenvolvidas por Lovrić 
e Osteryoung leva em consideração as condições apresentadas acima e um procedimento matemático que origina a corrente normalizada para a SWV, que é definida por um máximo de corrente, onde são considerados os potenciais de pico no valor do máximo de corrente $\left(E_{p}\right)$ e a largura de meia-altura da onda de redução e/ou oxidação $\left(\Delta E_{p / 2}\right)$. Desta maneira a corrente normalizada é apresentada como:

$\Phi=\frac{i \sqrt{\pi}}{n F q A^{*} \sqrt{2 f D_{A}}}$

onde $\Phi$ é a corrente normalizada, $i$ a corrente de pico, $n$ o número de elétrons, $F$ a constante de Faraday, $q$ a área do eletrodo, $A^{*}$ a concentração do reagente no seio da solução, $f$ a freqüência e $D_{A}$ o coeficiente de difusão do reagente.

Osteryoung e Lovrić realizaram cálculos teóricos, pela simulação de reações reversíveis, irreversíveis e quase-reversíveis. Para estas simulações, um algoritmo COOL (assim chamado em função das pessoas que o desenvolveram, Chernoff, O'Dea, Osteryoung e Lane) foi utilizado, o que possibilitou todo o tratamento teórico da SWV. A teoria desenvolvida por eles foi testada e aplicada em sistemas com comportamento eletroquímico já conhecido, tais como azul de metileno, midazolam, berberina e alguns metais como $\mathrm{Cd}$ e $\mathrm{Pb}^{16-19}$.

Os cálculos teóricos demonstraram que as características dos voltamogramas teóricos $\left(\mathrm{i}_{\mathrm{p}}, E_{p}\right.$ e $\left.\Delta E_{p / 2}\right)$ dependem linearmente de alguns parâmetros utilizados na SWV e do tipo de sistema redox. De acordo com estes cálculos, observou-se que a forma do voltamograma na SWV pode ser normalizado em função da razão entre a corrente de pico $\left(i_{p}\right)$ e a largura de meia altura do pico de potencial $\left(\Delta E_{p / 2}\right)$, e que o potencial de pico depende inteiramente do produto $\alpha n$. Com isto, eles puderam observar que, mantendo-se constante um parâmetro e variando-se todos os outros, poder-se-ia estudar as relações de proporcionalidade entre cada uma das variáveis e seu comportamento em cada tipo de sistema em estudo ${ }^{13}$.

De acordo com a teoria da SWV, a magnitude, perfil e posição dos picos redox dependem do grau de reversibilidade e do valor do coeficiente de transferência eletrônica da espécie eletroativa, sendo que o caráter da reação redox pode causar um aumento ou diminuição significativa na resposta obtida ${ }^{17}$.

Sendo assim, a avaliação das respostas obtidas, após a definição dos parâmetros envolvidos na técnica, torna possível a interpretação dos resultados e a definição do tipo de reação envolvida em cada sistema estudado. Além do mais, ainda é possível obter informações sobre o mecanismo e a cinética dos processos eletródicos de interesse.

\section{Sistemas reversíveis}

Se não ocorre adsorção da espécie eletroativa na superfície do eletrodo e se o coeficiente de transferência de carga é considerado $0,5(\alpha=0,5)$, então a intensidade da corrente de pico para sistemas reversíveis é, no mínimo, duas vezes maior que para sistemas totalmente irreversíveis, o que mostra uma maior sensibilidade da SWV para sistemas reversíveis ${ }^{22}$. Isto se deve ao fato de que a corrente resultante é a diferença entre as correntes diretas e inversas, e quanto maior a reversibilidade da reação maior é a contribuição da corrente inversa, aumentando significativamente a corrente resultante e, conseqüentemente, a resposta em termos de intensidade de corrente.

$I_{t}=I_{d}-I_{i}$

onde $I_{d}$ é a corrente direta e $I_{i}$ é a corrente inversa.

Se o produto não adsorve na superfície do eletrodo de trabalho, a resposta para sistemas reversíveis pode chegar a ser até cinco vezes maior que se houvesse adsorção ${ }^{22}$.
A corrente de pico normalizada $\left(\Phi_{p}\right)$ aumenta proporcionalmente com o número de elétrons, mas este aumento não é linear. Se produto e reagente adsorvem à superfície do eletrodo de trabalho, o valor de $\Phi_{p}$ é independente da variação no incremento de varredura de potencial e do coeficiente de transferência eletrônica $(\alpha)$. No entanto, se apenas o reagente sofre adsorção, a relação entre a corrente de pico normalizada e o incremento de varredura é linear, independente da reversibilidade da reação redox $\left(\Phi_{p}=k \Delta E_{s}\right)$, onde $k$ é um fator de proporcionalidade que depende da cinética da reação pela relação da constante de velocidade com a freqüência de aplicação dos pulsos de potencial $\left(k_{r} / 2 f\right)$ :

$\Phi=\frac{k_{r}}{2 f} \Delta E_{s}$

De acordo com O'Dea e Osteryoung ${ }^{16}$, um outro critério para demonstrar a reversibilidade da reação redox, é a análise da constante cinética. A razão entre este fator cinético e a frequiência de aplicação dos pulsos de potencial é um importante parâmetro utilizado em estudos cinéticos com a SWV. Para uma reação redox reversível, o logaritmo da constante de velocidade da reação em função do dobro da freqüência é dado por:

$\log k_{r} / 2 f \geq 0$ (adsorção de reagente)

$\log k_{r} / 2 f=0,5$ (adsorção de reagente e produto)

Com relação ao comportamento da corrente de pico $\left(I_{p}\right)$ em função da variação da frequiência de aplicação dos pulsos de potenciais $(1 / \tau)$, observa-se que, para sistemas reversíveis, o aumento da corrente é proporcional à raiz quadrada da frequiência:

$I_{p}=k_{r} f^{1 / 2}$

$\mathrm{O}$ aumento da freqüência de aplicação dos pulsos de potencial causa um aumento na resposta de corrente, com a conseqüente melhoria na sensibilidade da análise. Contudo, este aumento na frequiência deve ser observado com atenção, já que a reversibilidade da reação pode diminuir e, assim, a corrente de pico passa a crescer exponencialmente, o que deve ser devidamente considerado numa curva de analítica.

A relação entre a corrente de pico e a raiz quadrada da freqüência é linear, considerando-se sistemas reversíveis. No entanto, pequenos desvios da linearidade podem surgir quando se trabalha com valores altos de freqüência (maiores que $1000 \mathrm{~Hz}$ ) em função da resistência da própria solução a uma velocidade de varredura tão elevada. Para freqüências inferiores a $30 \mathrm{~Hz}$, desvios da linearidade também podem surgir devido a ruídos provenientes do circuito eletrônico e que, em velocidades tão baixas, interferem mais intensamente nas respostas voltamétricas. Equações para corrente de pico são dadas por:

$$
\begin{array}{ll}
I_{p}=\text { const. } n^{x} \Delta E_{s} f \Delta E_{p}{ }^{y} t_{0}{ }^{1 / 2} A^{*} & \text { para } 1<\mathrm{x}<2 ; \mathrm{y}<1 \\
I_{p}=\text { const. } n^{x} f^{1 / 2} \Delta E_{p}{ }^{y} t_{0}{ }^{1 / 2} A^{*} & \text { para } 1<\mathrm{x}<2 ; \mathrm{y}<1
\end{array}
$$

onde a Equação (7) fornece a corrente de pico para reações reversíveis com apenas o reagente adsorvido e a Equação (8), com adsorção de produtos e reagentes.

Os potenciais de pico apresentam uma importante relação com a variação da frequiência, já que com estes dados é possível se obter informações sobre o número de elétrons envolvidos no processo redox e, até mesmo, do coeficiente de transferência eletrônica para reações não simétricas $(\alpha \neq 0,5)$. 
Para reações reversíveis, o potencial de pico apresenta uma relação linear com o logaritmo da frequiência de aplicação dos pulsos de potencial, apresentando uma inclinação de:

$$
\Delta E / \Delta \log (f)=-2,3 R T / 2 n F
$$

para a adsorção do reagente. Se reagente e produto adsorvem, a reação redox reversível, neste caso, é independente da frequiência de aplicação dos pulsos de potenciais.

A largura de meia altura $\left(\Delta E_{p / 2}\right)$ é um outro parâmetro considerado na análise de dados obtidos com SWV, podendo também ser utilizada para se avaliar a reversibilidade de reações redox ${ }^{14}$. Ela é independente do incremento de varredura de potenciais e do tempo inicial, mas depende da amplitude dos pulsos de potencial aplicados $\left(\Delta E_{p}\right)$ e da constante cinética da reação $\left(k_{r} / 2 f\right)$.

A largura de meia altura para uma reação reversível é independente da amplitude $\left(\Delta E_{p}\right)$ e da freqüência de aplicação dos pulsos de potencial, mas o produto $n \Delta E_{p / 2}$ depende, de maneira não linear, do número de elétrons transferidos e da amplitude da onda. Sob a influência de adsorção, a largura de meia altura é dada por $^{22}$ :

$\Delta E_{p / 2}=101 / n$ (adsorção do reagente)

$\Delta E_{p / 2}=97 / n$ (adsorção de produto e reagente)

A amplitude de aplicação dos pulsos de potencial, também influencia nos valores obtidos para a corrente de pico, de maneira linear se $\Delta E P=20 / n \mathrm{mV}$.

Para reações reversíveis com adsorção de produto e reagente, a corrente de pico aumenta proporcionalmente somente para valores de amplitude menores que $60 \mathrm{mV}$, valores de amplitudes de aplicação dos pulsos de potencial maiores, provocam uma mudança na largura de meia-altura $\left(\Delta E_{p / 2}\right)$ influenciando a resposta voltamétrica.

\section{Sistemas irreversíveis}

Para sistemas onde ocorram reações totalmente irreversíveis, a corrente de pico $\left(I_{p}\right)$ é até cinco vezes menor que a corrente de pico obtida de um sistema reversível. O valor de $I_{p}$ é menor devido à menor contribuição proveniente da corrente inversa, com o valor da corrente resultante praticamente coincidente com o da corrente direta. A corrente de pico é dada por:

$$
I_{p}=\text { const. } \alpha n^{2} \Delta E_{s} f \Delta E_{p}{ }^{x} t_{0}^{1 / 2} A^{*}
$$

A corrente de pico normalizada $\left(\Phi_{p}\right)$, para reações redox totalmente irreversíveis apresenta uma dependência linear com o número de elétrons transferidos durante o processo:

$$
\Phi_{p}=k \alpha n \Delta E
$$

Esta linearidade depende de algumas condições de contorno, ou seja, considerando-se a transferência de até quatro elétrons $(n \leq 4)$, incremento de varredura menor que $25 \mathrm{mV}$ dividido pelo número de elétrons transferidos $(\Delta E S \leq 25 / n \mathrm{mV})$ e o coeficiente de transferência eletrônica $\alpha=0,5$.

A corrente de pico $(I)$ apresenta uma relação linear com a frequiência de aplicação do pulso de potencial, se a reação é totalmente irreversível:

$$
I_{p}=k_{r} f
$$

O aumento da freqüência de aplicação dos pulsos de potenciais pode ser um dos artifícios utilizados para se melhorar a resposta da
SWV, já que se todas as outras variáveis forem fixadas, a sensibilidade é dada em função apenas da frequiência da onda. Uma outra forma de melhorar a sensibilidade analítica na SWV com processos redox totalmente irreversíveis é aumentar a amplitude de aplicação dos pulsos de potenciais, isto porque, para valores de amplitudes maiores que $20 \mathrm{mV}$, a largura de meia-altura $\left(\Delta E_{p / 2}\right)$ se mantêm constante.

$\mathrm{O}$ aumento do incremento de potencial $\left(\Delta E_{p}\right)$ também melhora a sensibilidade analítica da SWV, de maneira semelhante ao aumento da amplitude de aplicação dos pulsos de potência.

Para os potenciais de pico em reações redox totalmente irreversíveis, existe uma relação linear entre $\left(E_{p}\right)$ e o logaritmo da frequiência, com uma inclinação de:

$\Delta E / \Delta \log f=-2,3 R T / \alpha n F$

ou $-59 / \alpha n \mathrm{mV}$ por década. Esta relação é utilizada para se estimar o número de elétrons envolvidos na reação redox e o coeficiente de transferência eletrônica em reações não simétricas.

Para reações totalmente irreversíveis, a largura de meia-altura $\left(\Delta E_{p / 2}\right)$ é independente da amplitude dos pulsos se $\Delta E_{p}>20 \mathrm{mV}$ para adsorção do reagente e $\Delta E_{p}>40 \mathrm{mV}$ para adsorção do produto e reagente. Sendo assim, é possível se considerar que:

$\Delta E_{p / 2}=\frac{(63,5 \pm 0,5)}{\alpha n}$

de maneira que para um valor de $\alpha=0,5$ o $\Delta E_{p / 2}$ é igual a $127 / n$.

Um critério cinético que pode ser utilizado para determinar a irreversibilidade de um processo redox é o comportamento da constante cinética da reação $\left(k_{r}\right)$ em função da variação da freqüência, onde se observa que para um processo totalmente irreversível:

$\log k_{r} / 2 f \leq-5$

\section{Sistemas quase-reversíveis}

Em uma reação quase-reversível, a corrente de pico normalizada $\left(\Phi_{P}\right)$ aumenta em função do aumento da constante de velocidade da reação $\left(k_{r}\right)$ e com o número de elétrons transferidos no processo redox, mas este aumento não é linear. Além disso, ela independe da frequiência de aplicação dos pulsos de potencial.

A variação no coeficiente de transferência eletrônica $(\alpha)$ altera, de maneira significativa, o grau de reversibilidade em um sistema quase-reversível sendo que o aumento de $\alpha$ induz um comportamento análogo na intensidade da corrente de pico normalizada, como se a velocidade da reação estivesse sendo aumentada ${ }^{18}$.

Em geral, em um sistema quase-reversível, a corrente de pico normalizada e o coeficiente de transferência eletrônica exibem um máximo e, após este máximo, o valor da corrente de pico normalizada decresce de maneira não linear com o aumento do coeficiente de transferência eletrônica ${ }^{19}$.

Em um processo redox quase-reversível, a intensidade de corrente de pico, que pode determinar a sensibilidade da técnica, aumenta em proporção à constante de velocidade da reação $\left(k_{r}\right)$ e não apresenta relação linear com a freqüência de aplicação dos pulsos de potencial, mas pode ser avaliada em função do parâmetro cinético crítico $(k)$.

Analisando-se a resposta da corrente de pico em função da freqüência de aplicação dos pulsos de potencial, obtêm-se um máximo que pode ser usado para se obter o valor exato da constante cinética do processo redox:

$k_{r}=k_{\max } \cdot f_{\max }$ 
A constante cinética depende do valor de $\alpha$ e de $\Delta \mathrm{E}_{\mathrm{p}} \cdot \mathrm{n}$, mas independe de $\Delta \mathrm{E}_{\mathrm{s}} \cdot \mathrm{n}$ e da concentração do reagente, quando não há adsorção do reagente. Quando o valor de $\alpha$ é desconhecido, ele pode ser determinado avaliando-se o valor de $k$ no máximo da relação $\Delta I_{p} / f$ versus $f$ :

$$
k_{\max }=1,13+1,13(\alpha-0,55)^{2}
$$

Os potenciais de pico também não apresentam nenhuma relação linear com a freqüência de aplicação dos pulsos de potencial.

A cinética para um processo quase-reversível, considerando-se os dados de SWV, mostra que a relação entre a constante cinética da reação e a freqüência de aplicação dos pulsos de potencial é dada por:

$\log k_{r} / 2 f=0,01 \quad$ (adsorção de reagente)

$\log k_{r} / 2 f>0,5$ (adsorção de reagente e produto)

A Tabela 1 apresenta um resumo para o comportamento da corrente de pico em função da variação da frequiência de aplicação dos pulsos de potencial, para cada um dos sistemas redox considerados.

A Tabela 2 apresenta um resumo das relações para as inclinações dos potenciais de pico com a freqüência de aplicação dos pulsos de potenciais, de acordo com cada tipo de sistema redox, e a Tabela 3 mostra um resumo com as equações matemáticas que englobam o modelo teórico para o SWV, desenvolvido por Osteryoung e Lovrić, aplicadas a eletrodos convencionais e eletrodos de mercúrio com gota estática.
Tabela 1. Relação de correntes de pico e freqüência de aplicação dos pulsos de potencial $(f)$, de acordo com cada sistema, sendo $k$ a constante de velocidade da reação

\begin{tabular}{ll}
\hline Sistema & Relação \\
\hline Irreversível & $I_{p}=k f$ \\
Reversível & $I_{p}=k f^{1 / 2}$ \\
Quase-Reversível & Não linear \\
\hline
\end{tabular}

Tabela 2. Inclinação das relações lineares para os potenciais de pico com a frequiência de aplicação dos pulsos de potenciais, de acordo com cada tipo de reação redox

\begin{tabular}{ll}
\hline Sistema & Inclinação \\
\hline Irreversível & $\frac{\partial E_{p}}{\partial \log (f)}=\frac{-2,3 R T}{\alpha n F}$ \\
Reversível, sem adsorção do produto & $\frac{\partial E_{p}}{\partial \log (f)}=\frac{-2,3 R T}{2 n F}$ \\
$\begin{array}{l}\text { Reversível, com adsorção do produto e } \\
\text { do reagente }\end{array}$ & Relação não linear \\
\hline
\end{tabular}

Tabela 3. Resumo dos valores de correntes de pico, potenciais de pico, largura de meia-altura e constante de velocidade para os vários sistemas redox, de acordo com Lovrić ${ }^{13,14,18,19,22}$ e Osteryoung $5,6,8,15,16$

\begin{tabular}{|c|c|}
\hline Sistema & Resultados \\
\hline $\begin{array}{l}\text { Reações redox totalmente irreversíveis } \\
\text { com reagente, ou reagente e produto } \\
\text { adsorvido }\end{array}$ & $\begin{array}{l}I_{p}=\text { const. } \alpha n^{2} \Delta E_{s} f \Delta E_{p}^{x} t_{0}^{1 / 2} A^{*} \quad \text { para } x<1 \\
\frac{\partial E_{p}}{\partial \log (f)}=\frac{-2,3 R T}{\alpha n F} \\
\Delta E_{p / 2}=\frac{(63,5 \pm 0,5)}{\alpha n} \\
\log \left(k_{r} / 2 f\right) \leq-5\end{array}$ \\
\hline $\begin{array}{l}\text { Reações redox reversíveis com } \\
\text { apenas o reagente adsorvido }\end{array}$ & $\begin{array}{l}I_{p}=\text { const. } n^{x} \Delta E_{s} f \Delta E_{p}{ }^{y} t_{0}^{1 / 2} A^{*} \quad \text { para } 1<\mathrm{x}<2 ; \mathrm{y}<1 \\
\frac{\partial E_{p}}{\partial \log (f)}=\frac{-2,3 R T}{2 n F} \\
E_{p / 2}=\frac{101}{n} \\
\log \left(k_{r} / 2 f\right) \geq 0\end{array}$ \\
\hline $\begin{array}{l}\text { Reações redox reversíveis com } \\
\text { produto e reagente adsorvidos }\end{array}$ & $\begin{array}{l}I_{p}=\text { const. } n^{x} f^{1 / 2} \Delta E_{p}^{y} t_{0}^{1 / 2} A^{*} \text { para } 1<\mathrm{x}<2 ; \mathrm{y}<1 \\
\frac{\partial E_{p}}{\partial \log (f)}=0 \\
E_{p / 2}=\frac{97}{n} \\
\log \left(k_{r} / 2 f\right)=0,5\end{array}$ \\
\hline
\end{tabular}




\section{Vantagens no uso da voltametria de onda quadrada}

Do ponto de vista prático, a maior vantagem da SWV é a possibilidade de se obter correntes de pico bem definidas em experimentos executados em alta velocidade de varredura, melhorando, assim, a sensibilidade da técnica. Além disto, por tratar-se de uma técnica de pulso, a corrente faradaica pode ser coletada em um intervalo de tempo adequado para que a contribuição da corrente capacitiva tenha se minimizado.

Quando se considera a utilização do eletrodo gotejante de mercúrio, a maior vantagem da SWV é a possibilidade de se promover uma varredura completa do potencial durante o tempo de vida de uma única gota de mercúrio, reduzindo consideravelmente o tempo necessário à realização do experimento, comparado com as tradicionais técnicas eletroanalíticas e a manipulação do mercúrio dispensado após o experimento.

Uma outra vantagem apresentada pela SWV é a redução no ruído de fundo por meio de varreduras repetitivas. Além disso, ainda é possível, pela observação dos sinais das varreduras direta e inversa, se obter as informações análogas àquelas obtidas utilizando-se a VC, porém com uma sensibilidade maior devido à minimização da contribuição da corrente capacitiva.

A velocidade efetiva para uma análise por SWV é dada em função do incremento de varredura de potencial e a frequiência de aplicação dos pulsos de potencial $(f \Delta E$ ). Sendo assim, tempos experimentais muito curtos podem ser obtidos utilizando-se freqüências moderadas, de maneira que os dados obtidos possam oferecer informações sobre a cinética do processo eletródico, já que habitualmente se sugerem velocidades de $2 \mathrm{~V} \mathrm{~s}^{-1}$ para estudos cinéticos, o que é facilmente obtido com a utilização da SWV.

\section{Contribuições à teoria da voltametria de onda quadrada}

Os modelos desenvolvidos por Lovrić e Osteryoung foram testados experimentalmente e aplicados para diversos sistemas, sob variadas condições experimentais. Contudo, alguns trabalhos surgiram com o intuito de contribuir para o completo estabelecimento da SWV como ferramenta de trabalho em eletroquímica e eletroanalítica.

A mudança de quaisquer parâmetros envolvidos na SWV pode influenciar intensamente a resposta final, tanto em sistemas reversíveis quanto em sistemas totalmente irreversíveis, com ou sem adsorção de produtos e/ou reagentes. Por isto, a utilização de sistemas conhecidos, tais como chumbo (sistema reversível) e níquel (sistema irreversível) possibilitaram a comprovação e aplicação dos modelos desenvolvidos por Lovrić e Osteryoung para sistemas inorgânicos ${ }^{21}$.

Outros metais, como tálio, cádmio, cobre, índio, bismuto e cobalto em meios contendo diversos eletrólitos suportes, também foram utilizados para se avaliar a aplicação dos modelos desenvolvidos, assim como a intensificação das correntes de pico em função da adsorção de produto ou reagente e a reversibilidade do processo redo ${ }^{22}$.

Os modelos propostos foram testados para compostos orgânicos com o objetivo de se explorar os tratamentos quantitativos possíveis e a aplicação da SWV, considerando-se reações envolvendo processos redox com materiais fortemente adsorvidos na superfície do eletrodo de trabalho ${ }^{23}$.

As respostas obtidas com a aplicação da SWV em sistemas redox mais complexos, em função dos processos cinéticos envolvidos com o eletrodo ou por reações químicas catalíticas foram consideradas por Lovrić, com a aplicação dos modelos em um sistema orgânico muito sensível à adsorção ${ }^{24}$.

Reações envolvendo transferência eletrônica lenta, reações de transferências eletrônicas precedidas e/ou seguidas por reações químicas, e reações químicas catalíticas foram consideradas, e curvas características de cada um destes processos foram utilizadas para o estudo de dados cinéticos em experimentos de SWV, de onde também, é possível avaliar-se o mecanismo de transferência eletrônica ${ }^{25}$.

Até o momento, todo o tratamento teórico apresentado leva em consideração apenas a utilização de eletrodos sólidos convencionais e eletrodos de mercúrio. Contudo, na SWV, assim como em outras técnicas, a resposta de corrente depende do tamanho e do tipo de eletrodo utilizado. Com isto, algumas contribuições importantes, envolvendo a utilização de outros tipos de eletrodos, surgiram, com o intuito de aplicar ou ampliar a utilização da SWV.

Uma importante contribuição foi feita por Miles e Compton ${ }^{26}$ que estenderam a teoria da SWV para a aplicação em eletrodos hidrodinâmicos, considerando-se sistemas eletroquimicamente reversíveis, irreversíveis e quase-reversíveis. Nas considerações de Miles e Compton, equações integrais complementares foram adicionadas em função do transporte de massa por convecção originada da utilização de eletrodos rotatórios.

Além de considerar a convecção presente na utilização de eletrodos rotatórios, Miles e Compton também avaliaram a resposta da SWV quando eletrodos tubulares (eletrodos encravados na parede do canal definido por uma junta entre dois blocos) e células de fluxo são utilizadas ${ }^{27}$. Neste tipo de eletrodo, o transporte de massa ocorre por um fluxo convectivo imposto ao eletrodo e o transporte de massa por difusão pode ser ignorado, o que leva a algumas considerações diferenciadas para os modelos propostos na SWV. Desta maneira, as propriedades voltamétricas da SWV são variadas em função da velocidade de fluxo, e a dependência da corrente diminui quando a frequiência de aplicação dos pulsos de potenciais é aumentada, no entanto, quando a freqüência é muito alta, a resposta deixa de depender da velocidade de fluxo.

Simulações numéricas gerais foram efetuadas, baseadas em métodos implícitos para experimentos de SWV em casos de sistemas reversíveis $^{28}$, irreversíveis ${ }^{29}$, quase-reversíveis ${ }^{30}$ e mecanismos EC e ECE. Cada tipo de processo foi considerado e as propriedades voltamétricas, como corrente de pico, potencial de pico e largura de meia-altura, foram consideradas para quantificar e identificar espécies eletroativas e fornecer informações cinéticas e mecanísticas quando eletrodos hidrodinâmicos e eletrodos hemisféricos são utilizados.

A substituição de eletrodos convencionais por ultramicroeletrodos, também necessitou de considerações especiais advindas do tipo de transporte de massa predominante nos ultramicroeletro$\operatorname{dos}^{32}$. Quando estes tipos de eletrodos são utilizados, a resposta voltamétrica é exclusivamente dependente da geometria do eletrodo e da velocidade do processo de transferência eletrônica. Assim, os critérios de reversibilidade não são apresentados em função da freqüência de aplicação dos pulsos de potenciais ${ }^{33-35}$.

Uma das mais recentes contribuições à teoria da SWV foi um estudo realizado por Mirceski ${ }^{36}$, que avaliou teoricamente, utilizando simulações no algoritmo COOL, a influência da queda ôhmica na resposta da SWV. Desta forma, foi avaliado que o grau de influência da queda ôhmica depende das condições experimentais (área do eletrodo, concentração da espécie eletroativa e temperatura da solução), da natureza da reação redox (número de elétrons envolvidos na transferência eletrônica e coeficiente de difusão das espécies eletroativas), da resistência ôhmica de diferentes fontes e da frequiência de aplicação dos pulsos de potenciais.

\section{Voltametria de múltipla onda quadrada}

$\mathrm{Na}$ voltametria de onda quadrada, um par de pulsos de potencial com sinais opostos é aplicado em cada degrau de potencial. Contudo, há ainda uma outra técnica eletroanalítica em que múltiplos pul- 
sos de potencial com amplitude constante são aplicados em um mesmo degrau, promovendo uma melhoria significativa na resposta de corrente e na relação resposta/ruído. Esta técnica é chamada de voltametria de múltipla onda quadrada $(\mathrm{MSWV})^{37}$ e é uma extensão da SWV.

Como na MSWV, múltiplos pulsos de potencial são aplicados em um mesmo degrau de potencial, as respostas de corrente-potencial são produzidas em tempos muito curtos, a partir da soma das cargas medidas durante cada semi-período em um mesmo degrau da escada de potencial versus o potencial do degrau.

Na MSWV as respostas de corrente-potencial contêm uma contribuição considerável da variação de potencial na forma de escada, enquanto que na SWV, a escada de potencial possui uma pequena influência sobre a resposta, se considerarmos baixas freqüências de aplicação dos pulsos de potencial. Na MSWV esta contribuição varia rapidamente quando um determinado número de perturbações é aplicado em cada degrau desta escada de potenciais. Desta maneira, quanto maior o número de perturbações dentro de um mesmo degrau, maior será a resposta em termos de corrente melhorando, desta maneira, a sensibilidade.

A voltametria de múltipla onda quadrada complementa a SWV e pode ser utilizada com sucesso na análise eletroanalítica direta de baixas concentrações, especialmente quando os reagentes e os produtos são solúveis e as técnicas de pré-concentração não são muito aplicáveis $^{38}$.

\section{AGRADECIMENTOS}

Os autores agradecem ao CNPq e à FAPESP (Proc. Nos. 99/ 07891 e 00/03540-6) pelos auxílios concedidos.

\section{REFERÊNCIAS}

1. Brainina, K. H.; Talanta 1987, 34, 41.

2. Skoog, D. A.; Leary, J. J.; Principles of Instrumental Analysis, Saunders College Publishing: USA, 1992.

3. Rupp, E. B.; Zuman, P.; J. Agric. Food Chem. 1992, 40, 2016.

4. Wightman, R. M.; Maloy, J. T.; Faulkner, L. R.; Osteryoung, R.A.; Anson, F. C.; Anal. Chem. 1982, 54, 698A.

5. Osteryoung, J.; Osteryoung, R.; Anal. Chem. 1985, 57, 101A.

6. Osteryoung, J.; Schreiner, M. M.; Crit. Rev. Anal. Chem. 1988, Supplement $1, S 1,19$.
7. Dahmen, E. A. M. F.; Electroanalysis (Theory and applications in aqueous and non-aqueous media in automated chemical control), Elsevier: Amsterdam, 1986.

8. Osteryoung, J. G.; O’dea, J.J. Em Electroanalytical Chemistry; Bard, A. J., ed.; Marcel Dekker: New York, 1982, p. 14.

9. Ramaley, L.; Krause Jr., M. S.; Anal. Chem. 1969, 41, 1362.

10. Ramaley, L.; Krause Jr., M. S.; Anal. Chem. 1969, 41, 1365.

11. Christie, J. H.; Turner, J. A.; Osteryoung, R. A.; Anal. Chem. 1977, 49, 1899.

12. J Christie, J. H.; Turner, J. A.; Osteryoung, R. A.; Anal. Chem. 1977, 49,1904.

13. Lovrić, M.; Komorsky-Lovric, S.; Murray, R. W.; Electrochim. Acta 1988, 33, 739 .

14. Lovrić, M.; Komorsky-Lovric, S.; J. Electroanal. Chem. 1988, $248,239$.

15. O'dea, J. J.; Ribes, A.; Osteryoung, J. G.; J. Electroanal. Chem. 1993, 345, 287.

16. O'dea, J. J.; Osteryoung, R. A.; Anal. Chem.1981, 53, 695.

17. Nuwer, M. J.; O’Dea, J. J.; Osteryoung, J.; Anal. Chim. Acta 1991, 251, 13.

18. Komorsky-Lovric, S.; Lovrić, M.; J. Electroanal. Chem. 1985, 40, 1781.

19. Komorsky-Lovric, S.; Lovrić, M.; J. Electroanal. Chem. 1995, 384, 115.

20. Fatouros, N.; Krulic; D.; J. Electroanal. Chem. 1998, 443, 262.

21. Zachowski, E. J.; Wojciechowski, M.; Osteryoung, J. G.; Anal. Chim. Acta 1986, 183, 47.

22. Lovrić, M.; Branica, M.; J. Electroanal. Chem. 1987, 226, 239.

23. Ribes, A. J.; Osteryoung, J. G.; J. Electroanal. Chem. 1990, 287, 125

24. Komorsky-Lovric; S., J. Electroanal. Chem. 2000, 482, 222.

25. Santos, M. M. C.; Gonçalves, M. L. S.; Romão, J. C.; J. Electroanal. Chem. 1996, 413,97

26. Miles, A. B, Compton; R. G.; J. Electroanal. Chem. 2000, 487, 75

27. Miles, A. B., Compton; R. G.; J. Electroanal. Chem. 2001, 499, 1.

28. Brookes, B. A.; Ball, J. C.; Compton, R. G.; J. Phys. Chem. B 1999, 103, 5289.

29. Brookes, B. A.; Compton, R. G.; J. Phys. Chem. B 1999, 103, 9020.

30. Brookes, B. A.; Macfie, G.; Compton, R. G.; J. Phys. Chem. B 2000, 104, 5331.

31. Miles, A. B.; Compton, R. G.; J. Phys. Chem. B 2000, 104, 5784.

32. Osteryoung, J. Em Microelectrodes: Theory and Applications, Montenegro, M. I.; Queirós, M., A.; Dasbach, J., ed.; Kluwer Academic Publishers: London, 1991, p. 139.

33. Komorsky-Lovric, S.; Lovric, M.; Bond, A. M.; Electroanalysis 1993, 5, 29.

34. Whelan, D. P.; O’Dea, J. J.; Osteryoung, J.; J. Electroanal. Chem. 1986, 202, 23.

35. Tallman, D. E.; Anal. Chem. 1994, 66, 557.

36. Mirceski, V.; Lvric, M.; J. Electroanal. Chem. 2000, 497, 114.

37. Fatourus, N.; Simonin, J. P.; Chevalet, J.; Reeves, R. M.: J. Electroanal. Chem. 1986, 213, 1

38. Krulic, D.; Fatourus, N.; Chevalet, J.; J. Electroanal. Chem. 1990, 287, 215 . 